\title{
Splenic Ly6Chi monocytes are critical players in dystrophic muscle injury and repair
}

\author{
Giuseppe Rizzo, Rosanna Di Maggio, Anna Benedetti, Jacopo Morroni, Marina Bouche, \\ and Biliana Lozanoska-Ochser \\ Department of Anatomical, Histological, Forensic and Orthopedic Sciences, Sapienza University of Rome, Rome, Italy.
}

\begin{abstract}
Dystrophic muscle is characterized by chronic injury and a steady recruitment of inflammatory Ly $6 \mathrm{C}^{\mathrm{hi}}$ monocytes. Recent studies have identified the spleen as the dominant reservoir of these cells during chronic inflammation. Here, we investigated the contribution of splenic Ly $6 \mathrm{C}^{\text {hi }}$ monocytes to dystrophic muscle pathology. Using the $\mathrm{mdx}$ mouse model of muscular dystrophy, we show that $\mathrm{Ly}_{6 \mathrm{C}} \mathrm{hi}^{\mathrm{i}}$ monocytes accumulate in great numbers in the spleen over the course of the disease. The chemokine receptor CCR2 was upregulated on Ly6C hi monocytes in $m d x$ spleen before disease onset, thereby enabling their recruitment to dystrophic muscle. Splenectomy performed before disease onset significantly reduced the number of Ly $6 \mathrm{C}^{\text {hi }}$ monocytes infiltrating dystrophic limb muscle. Moreover, in the absence of splenic Ly $6 C^{\text {hi }}$ monocytes there was a significant reduction in dystrophic muscle inflammation and necrosis, along with improved regeneration during early disease. However, during late disease, a lack of splenic Ly6C ${ }^{\text {hi }}$ monocytes adversely affected muscle fiber repair, due to a delay in the phenotypic shift of proinflammatory $F 4 / 80^{+}$Ly $6 C^{\text {hi }}{ }^{2}$ CD206 ${ }^{\text {lo }}$ to antiinflammatory $\mathrm{F} 4 / 80^{+} \mathrm{Ly} 6 \mathrm{C}^{\mathrm{l}} \mathrm{CD} 206^{+}$macrophages. Overall, we show that the spleen is an indispensable source of $\mathrm{Ly} \mathrm{CC}^{\mathrm{hi}}$ monocytes in muscular dystrophy and that splenic monocytes are critical players in both muscle fiber injury and repair.
\end{abstract}

Authorship note: GR and RDM contributed equally to this work. MB and $\mathrm{BLO}$ are co-senior authors.

Conflict of interest: The authors have declared that no conflict of interest exists.

Copyright: (c) 2020, American Society for Clinical Investigation.

Submitted: June 3, 2019

Accepted: December 18, 2019

Published: January 30, 2020.

Reference information: JCI Insight. 2020;5(2):e130807.

https://doi.org/10.1172/jci.

insight.130807.

\section{Introduction}

Duchenne muscular dystrophy (DMD) is a genetic disease in which lack of dystrophin leads to chronic muscle injury and inflammation (1). The immune response plays a critical role in the pathogenesis of $\mathrm{DMD}$, and both the innate and the adaptive immune response contribute to the formation of the dystrophic muscle infiltrate (2-5). Monocytes and macrophages constitute the major part of the inflammatory cell infiltrate in dystrophic muscle $(2,6)$. There are two types of monocytes in circulation, classical Ly6 $\mathrm{C}^{\text {hi }}$ monocytes, characterized by the expression of the chemokine receptor CCR2, and Ly6 $\mathrm{C}^{\text {lo }}$ or patrolling monocytes, which lack expression of CCR2 but express the fractalkine receptor $\mathrm{CX}_{3} \mathrm{CR} 1$ (7). The classical Ly6 $\mathrm{C}^{\text {hi }}$ monocytes, also known as inflammatory monocytes, are among the first innate immune cells to be mobilized from the bone marrow into the circulation and recruited to the site of tissue injury (8). Once inside the tissue, and depending on the cytokine milieu, they give rise to both inflammatory and antiinflammatory macrophages $(7,8)$. On the other hand, Ly6 $C^{\text {lo }}$ monocytes patrol the vasculature under steady state and participate in the resolution of inflammation (7-9). We recently described the kinetics of the early inflammatory cell infiltrate in lower limb muscle in the $m d x$ mouse model of muscular dystrophy and showed that Ly6 $\mathrm{C}^{\mathrm{hi}}$ monocytes begin to infiltrate muscle early during the prenecrotic stage of the disease, at 2 weeks of age (6). They reach a peak during the necrotic phase at 4 weeks and gradually decline by 12 weeks of age when antiinflammatory macrophages begin to predominate. In spite of their important role in dystrophic muscle pathology, little is known about the origin of Ly6 $\mathrm{C}^{\text {hi }}$ monocytes in dystrophic muscle. Although the bone marrow has traditionally been considered as the major source of monocytes, there is now compelling evidence suggesting that the spleen is also an important reservoir of Ly6 $\mathrm{C}^{\text {hi }}$ monocytes and that the bone marrow likely outsources their production to the spleen in diverse disease settings (10). Thus, splenic monocytes were found to contribute to miocardial healing following ischemia, the formation of atherosclerotic lesions, and to disease progression in ALS (10-12). However, the contribution of splenic monocytes to dystrophic muscle pathology remains to be elucidated.

In this study we used the $m d x$ mouse model of muscular dystrophy to investigate whether splenic Ly $6 \mathrm{C}^{\text {hi }}$ monocytes participate in muscle injury, inflammation, and regeneration. We show for the first time 
to our knowledge that $\mathrm{Ly} 6 \mathrm{C}^{\text {hi }}$ monocytes accumulate in significant numbers in the spleens of $m d x$ mice and are recruited to dystrophic muscle where they contribute to both muscle fiber injury and repair.

\section{Results}

Ly6 hi monocytes accumulate in the spleens of mdx mice over the course of the disease. We previously showed that Ly $6 \mathrm{C}^{\text {hi }}$ monocytes begin to infiltrate $m d x$ limb muscle before the onset of overt disease at 2 weeks of age and reach a peak at 4 weeks of age when necrosis becomes evident (6). We therefore hypothesized that if the spleen represents an important source of Ly6C $\mathrm{Chi}^{\text {hi }}$ monocytes in the context of dystrophic muscle injury, then we should be able to detect changes in their number and or phenotype. To examine this hypothesis, we analyzed the number of Ly6C $\mathrm{Ch}^{\text {hi }}$ monocytes in the spleens of $m d x$ and WT mice at 1, 2, 4, and 12 weeks of age. $\mathrm{Ly} 6 \mathrm{C}^{\text {hi }}$ monocytes were identified by flow cytometry as $\mathrm{CD} 11 \mathrm{~b}^{+} \mathrm{F} 4 / 80^{\mathrm{lo}} \mathrm{Ly} 6 \mathrm{~g}^{\mathrm{lo}} \mathrm{CCR} 2^{+} \mathrm{Ly} 6 \mathrm{C}^{\mathrm{hi}}$ cells (Figure 1A). Interestingly, while there were no differences at 1 week of age, as one might expect, we observed a striking increase in the number of Ly6C $\mathrm{Ch}^{\text {hi }}$ monocytes at 2 weeks of age in $m d x$ spleens compared with WT spleens, and this significant increase was sustained at 4 weeks of age and gradually declined by 12 weeks of age when their numbers were comparable to those of WT spleens (Figure 1B). We also noticed an increase in Ly6g ${ }^{\text {hi }}$ neutrophils in the spleens of $m d x$ mice, and this will be the subject of future investigations. In support of these data, we found a significant increase in the frequency and number of Ly6 $\mathrm{C}^{\text {hi }}$ monocytes positive for the proliferation marker Ki-67 (Figure 1, C-E) and an increase in the expression level of mRNA for myeloid cell cytokines IL-1 $\beta$ and GM-CSF in $m d x$ spleens at 2 weeks of age (Supplemental Figure 1, B and C; supplemental material available online with this article; https://doi.org/10.1172/jci.insight.130807DS1). Of note, we observed increased frequency and number of myeloid progenitor cells in $m d x$ spleen (Supplemental Figure 2 , $\mathrm{A}-\mathrm{C})$, which likely give rise to Ly6 $\mathrm{C}^{\mathrm{hi}}$ monocytes as previously described $(12,13)$. Furthermore, a flow cytometric analysis of splenic Ly $6 \mathrm{C}^{\mathrm{hi}}$ monocytes in $m d x$ mice at 2 weeks of age revealed an upregulated expression of the chemokine receptor CCR2 (Figure 1, F and G), suggesting an activated status and a preparedness to be recruited and extravasate from circulation into the site of tissue injury.

These results show that there is a marked expansion of the splenic reservoir of Ly6 $\mathrm{C}^{\text {hi }}$ monocytes in $m d x$ mice before the onset of disease, suggesting a possible role in dystrophic muscle inflammation.

Splenectomy reduces the number of Ly6C $C^{\text {hi }}$ monocytes/macrophages infiltrating dystrophic muscle. In order to investigate the contribution of the splenic reservoir of $\mathrm{Ly} 6 \mathrm{C}^{\mathrm{hi}}$ monocytes to dystrophic muscle inflammation we splenectomized $m d x$ mice at 2 weeks of age, which is the time when Ly6C ${ }^{\text {hi }}$ monocytes begin to infiltrate muscle (6). We then performed analyses 2 weeks later, at 4 weeks of age, when typically $m d x$ muscle inflammation and damage peak; at 8 weeks of age when inflammation begins to subside; and at 12 weeks of age, when inflammation is minimal (ref. 6 and Figure 2A). Upon recruitment, Ly $6 \mathrm{C}^{\text {hi }}$ monocytes give rise to proinflammatory $\mathrm{F} 4 / 80^{+} \mathrm{CCR} 2^{+} \mathrm{Ly} 6 \mathrm{C}^{\mathrm{hi}}$ macrophages (or recently recruited monocytes/macrophages $[\mathrm{Mo} / \mathrm{MP}]$ ), which over time lose the expression of Ly6C and CCR2 and differentiate into antiinflammatory or proregenerative $\mathrm{F} 4 / 80^{+} \mathrm{CCR} 2-\mathrm{Ly} 6 \mathrm{C}^{\text {lo }}$ macrophages (Figure $2 \mathrm{~B}$ and refs. $6,7,14)$. Splenectomy significantly reduced the number of Ly6 $\mathrm{C}^{\text {hi }} \mathrm{Mo} / \mathrm{MP}$ in $m d x$ limb muscle at 4 (Figure 2, C and F) and 8 weeks of age (Figure 2, D and F). No significant differences were observed at 12 weeks of age (Figure 2, E and F). While there was no difference in the kinetics of Ly6 $\mathrm{C}^{\text {hi }}$ $\mathrm{Mo} / \mathrm{MP}$ in dystrophic muscle at 4, 8, and 12 weeks, the number of Ly6 $\mathrm{C}^{\text {hi }}$ monocytes was consistently lower in splenectomized $m d x$ mice compared with that in controls (Figure $2 \mathrm{~F}$ ). The expression of CCR2 by the recently recruited $\mathrm{F} 4 / 80^{+} \mathrm{Ly} 6 \mathrm{C}^{\mathrm{hi}} \mathrm{Mo} / \mathrm{MP}$ in muscles of $m d x$ control and splenectomized mice at 4 weeks of age was similar (Figure $2 \mathrm{G}$ ). Concomitant with the reduction in the number of inflammatory Mo/MP, the ligand for the CCR2 chemokine receptor, CCL2, which drives the recruitment of monocytes into the site of tissue injury (8), and the GM-CSF cytokine, which drives the expansion and activation of monocytes (15), were significantly reduced in limb muscle of splenectomized $m d x$ mice compared with controls (Figure 2, $\mathrm{H}$ and I).

Overall, these data suggest that the spleen serves as the dominant reservoir of Ly6 $\mathrm{Ch}^{\mathrm{hi}}$ monocytes during the acute phase of dystrophic muscle inflammation.

In the absence of the spleen, bone marrow increases the production of Ly6C hi monocytes. Our and previous findings suggest that the bone marrow outsources the supply of inflammatory monocytes to the spleen during chronic inflammation $(12,13)$. Therefore, we reasoned that in the absence of the spleen, the bone marrow might compensate by increasing the production of Ly6 $\mathrm{C}^{\text {hi }}$ monocytes. Indeed, 2 weeks after splenectomy, at 4 weeks of age, the number of Ly $6 \mathrm{C}^{\text {hi }}$ monocytes in the bone marrow of splenectomized $m d x$ mice was 
A

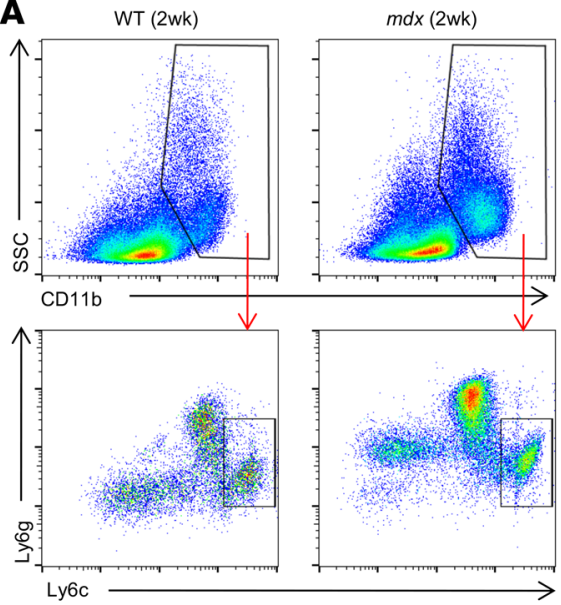

C

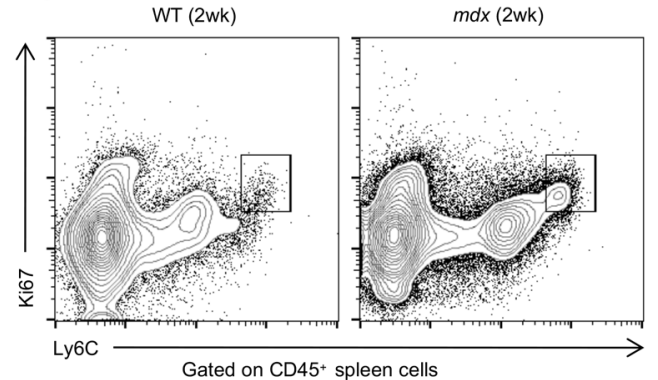

$\mathbf{F}$

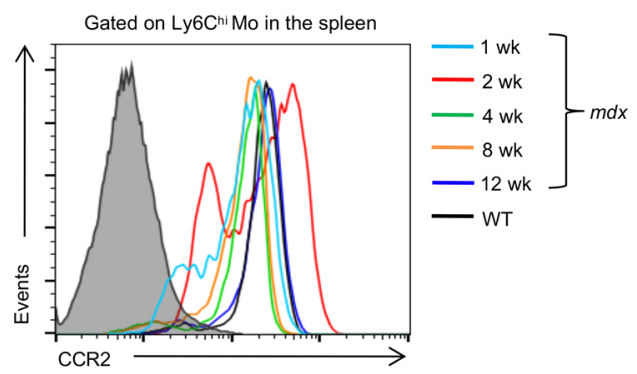

B

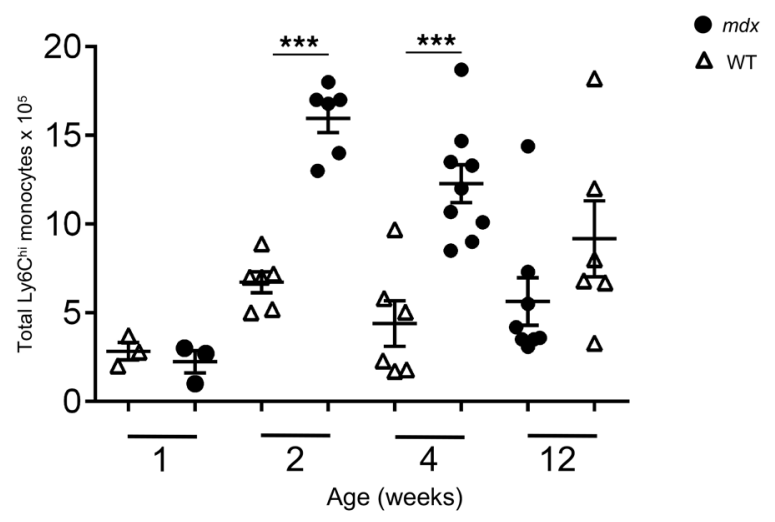

D

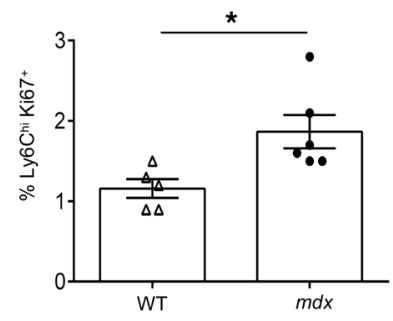

E

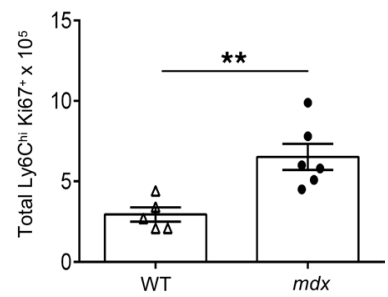

G

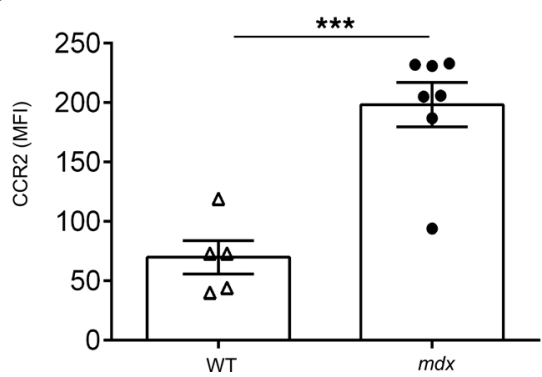

Figure 1. Expansion of the Ly6 $\mathbf{C}^{\text {hi }}$ monocyte reservoir in $\mathbf{m d x}$ spleen. The number and kinetics of Ly $6 \mathrm{C}^{\text {hi }}$ monocytes in $m d x$ and WT spleen were analyzed by flow cytometry at 1, 2, 4, and 12 weeks of age. (A) Representative flow cytometry plots showing the gating strategy for the identification of Ly6Chi monocytes. Ly6C $C^{\text {hi }}$ monocytes in the spleen were identified as CD45+CD11b+Ly6gloCCR2+Ly6C hi cells. (B) Quantification and kinetics of Ly6C ${ }^{\text {hi }}$ monocytes in $m d x$ and WT spleen at $1(n=3, m d x$ and $n=3$, WT), $2(n=6, m d x$ and $n=6$, WT), $4(n=9$, $m d x$ and $n=6$, WT), and $12(n=8, m d x$ and $n=6$, WT) weeks of

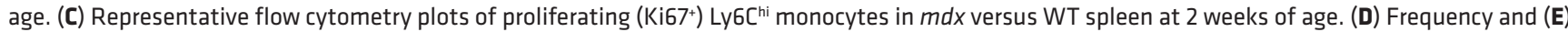
total number of proliferating Ki67+Ly6Chi monocytes in $m d x$ compared with WT spleen $(n=6, m d x$ and $n=5$, WT) at 2 weeks of age. (F) Representative histogram overlays of flow cytometric analysis of CCR2 receptor expression by Ly6Chi monocytes in mdx spleen at 1, 2, 4, 8, and 12 weeks and WT spleen at 2 weeks of age. (G) Mean fluorescence intensity (MFI) of CCR2 expression by Ly6C ${ }^{\text {hi }}$ monocytes in $m d x(n=7)$ and WT $(n=5)$ spleen, as determined by flow cytometric analysis at 2 weeks of age. Data are presented as mean $\pm \mathrm{SEM} .{ }^{*} P<0.05{ }^{* *} P<0.001 ;{ }^{* *} P<0.001$ by Student's $t$ test.

significantly increased compared with that of controls (Figure 3A). Intriguingly, the number of Ly6 $\mathrm{C}^{\mathrm{h}}$ monocytes was also increased in the bone marrow of splenectomized WT mice compared with that in controls, suggesting that the spleen might be a source of monocytes also in the steady state (Figure 3B). The expression of CCR 2 on bone marrow Ly $6 \mathrm{C}^{\text {hi }}$ monocytes was slightly increased in both splenectomized $m d x$ and WT mice compared with controls (Figure 3C). On the other hand, there was a modest increase in the number of circulating Ly6 $\mathrm{C}^{\text {hi }}$ monocytes in splenectomized compared with control $m d x$ mice (Figure 3D) and a significant increase in splenectomized compared with control WT mice (Figure 3E). Similar to bone marrow, there was a small increase in the expression of CCR2 by Ly6 $\mathrm{C}^{\mathrm{hi}}$ monocytes in splenectomized compared with control $m d x$ mice (Figure $3 \mathrm{~F}$ ). These results show that the bone marrow increases the production of Ly6C $\mathrm{C}^{\text {hi }}$ monocytes following splenectomy, but, as shown in Figure 2, fails to fully compensate for the absence of splenic Ly6 $\mathrm{C}^{\text {hi }}$ monocytes. 

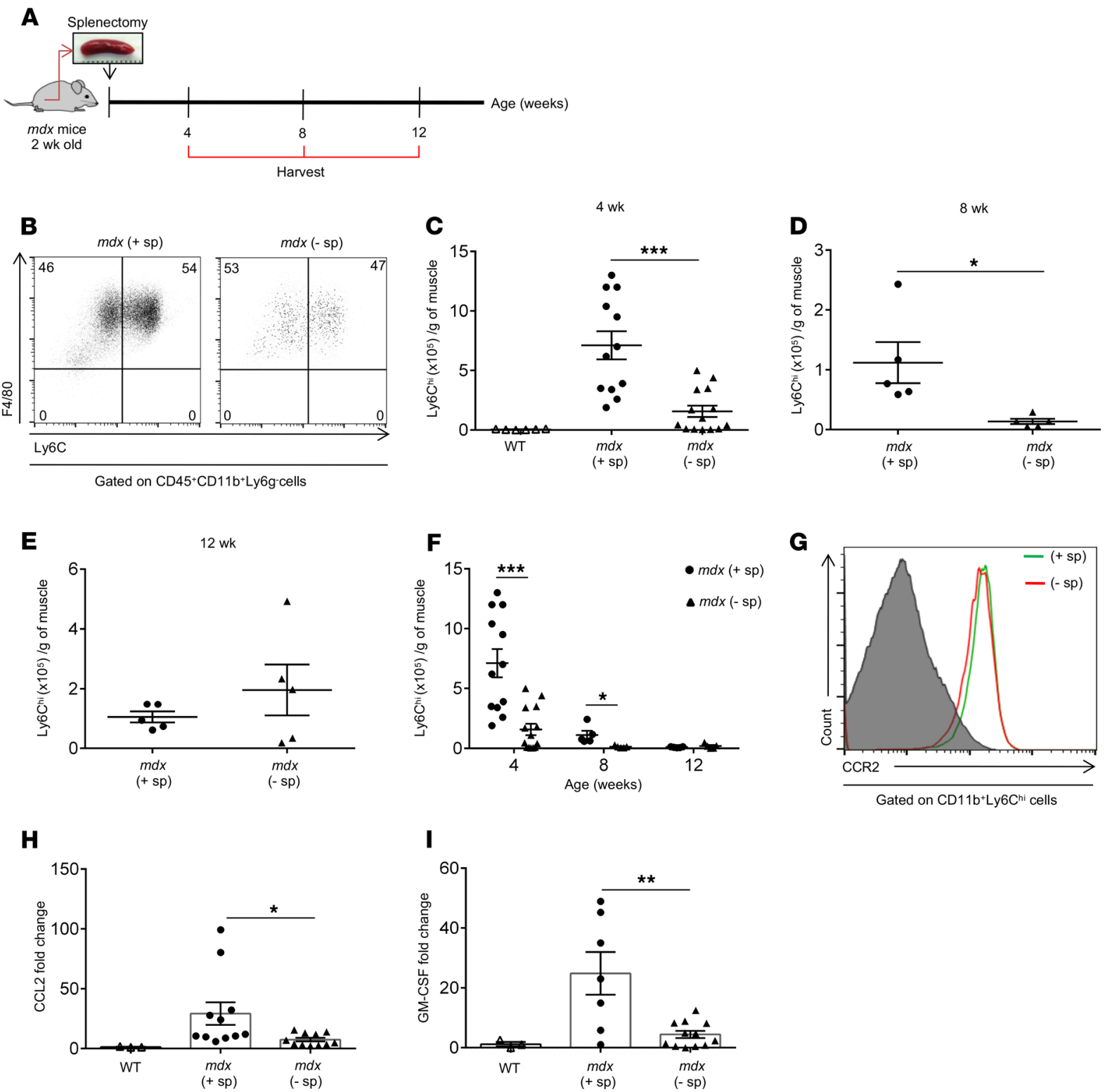

Figure 2. Splenectomy reduces the number of Ly $6 \mathrm{C}^{\text {hi }}$ monocytes recruited to dystrophic muscle. (A) Schematic presentation of the experimental protocol. Mice were splenectomized at 2 weeks of age and allowed to recover for at least 2 weeks before analysis. Control mice were treated the same way but without removal of the spleen. Following splenectomy the Ly6C ${ }^{\text {hi }}$ monocyte infiltrate in hind limb muscle was analyzed by flow cytometry at 4,8 , and 12 weeks of age. (B) Representative flow cytometry plots of Ly6Chi monocytes in control (+ sp) and splenectomized (- sp) mdx muscle. Ly6Chi monocytes in muscle were identified as CD45+CD11b+'Ly6g-F4/80+CCR2+'Ly6Chi cells. (C) Quantification of Ly6Chi monocytes in dystrophic muscle of control and splenectomized $m d x$ mice at 4 weeks of age normalized to muscle mass ( $n=12$, control and $n=14$, splenectomized), (D) 8 weeks of age ( $n=5$ for both groups), and (E) 12 weeks of age ( $n=5$ for both groups). (F) Summary graph showing Ly6 $\mathrm{Chi}^{\mathrm{i}}$ monocyte kinetics in muscle of control and splenectomized mdx mice. (G) Expression of the CCR2 chemokine receptor in muscle of control and splenectomized $m d x$ mice at 4 weeks of age, analyzed by flow cytometry and presented as histogram overlays. (H) Expression levels of CCL2 and (I) GM-CSF mRNA in muscle of control and splenectomized mdx mice at 4 weeks of age, assessed by quantitative real-time PCR $\left(n=3, \mathrm{WT} ; n=11, m d x\right.$ control; $n=11, m d x$ splenectomized). Data are presented as mean $\pm \mathrm{SEM}$. ${ }^{*} P<0.05 ;{ }^{*} P<$ $0.01 ;{ }^{* *} P<0.001$ by 1 -way ANOVA (C, F, H, and I) and Student's $t$ test (D).

Lack of splenic Ly6C $C^{\text {hi }}$ monocytes is associated with a reduction in inflammation and early muscle fiber necrosis. Inflammatory Mo/MP contribute to early inflammation-mediated muscle damage and facilitate the recruitment of other inflammatory cells (2). Thus, removal of the splenic reservoir of Ly6 $\mathrm{C}^{\text {hi }}$ monocytes was associated with a significant reduction of the inflammatory infiltrate in muscle of splenectomized $m d x$ mice compared with controls (Figure 4, A and B). The total number of mononuclear cells isolated from limb muscle was significantly reduced in splenectomized $m d x$ mice compared with that in controls at all ages analyzed (Supplemental Figure 3, A-C). In addition, the number of $\mathrm{CD} 45^{+}$cells was significantly 
A

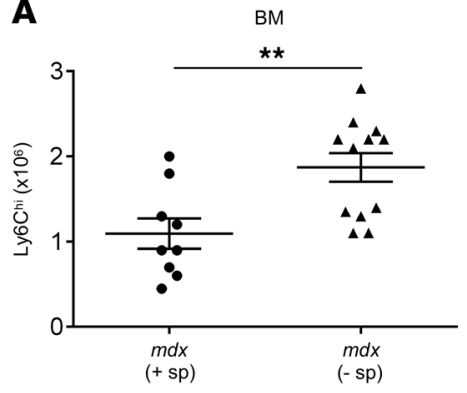

D

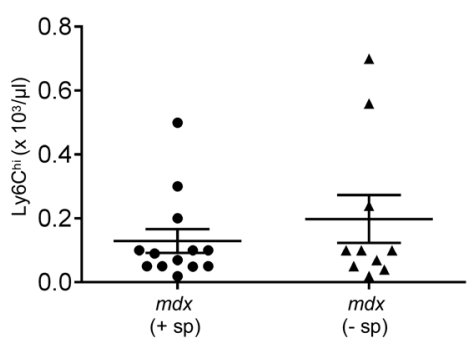

B

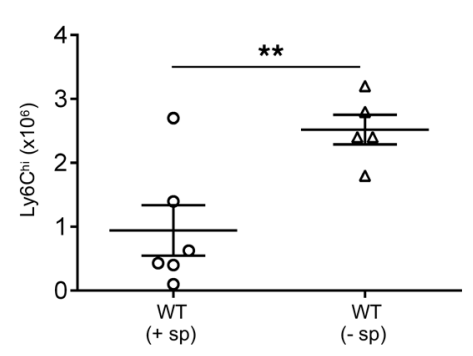

E

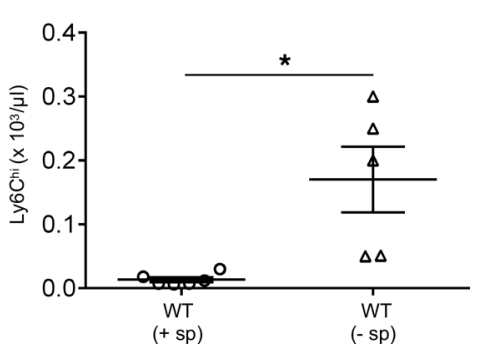

C

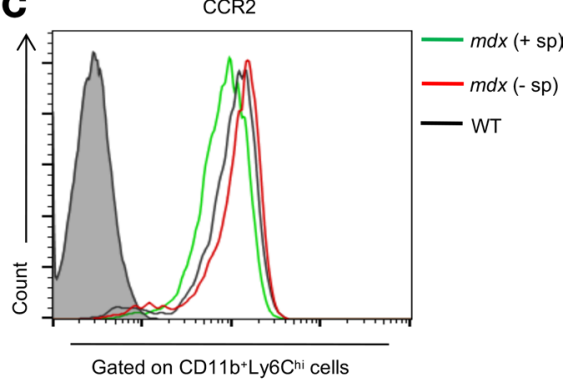

$\mathbf{F}$

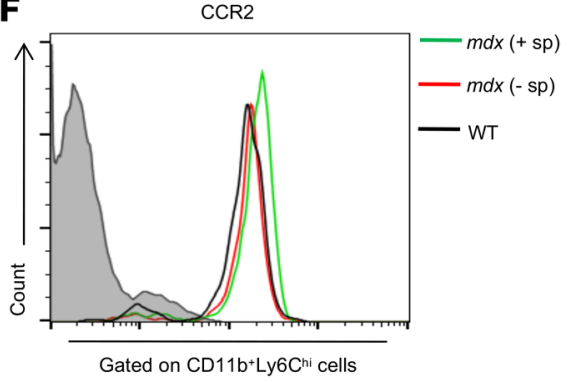

Figure 3. Effect of splenectomy on Ly $6 \mathrm{C}^{\text {hi }}$ monocyte numbers in the bone marrow and blood. Following splenectomy at 2 weeks of age Ly6C ${ }^{\text {hi }}$ monocytes in the bone marrow were analyzed by flow cytometry at 4 weeks of age. (A) Quantification of Ly6Chi monocytes in the bone marrow (per leg) of control (+ sp) and splenectomized ( $\mathrm{sp}) m d x(n=9, m d x$ control; $n=12, m d x$ splenectomized) and (B) WT mice ( $n=6$, WT control; $n=5$ WT splenectomized) by flow cytometry. (C) Histogram overlay of CCR2 expression on Ly6C ${ }^{\text {hi }}$ monocytes in the bone marrow of control and splenectomized mdx mice analyzed by flow cytometry. (D) Quantification of Ly6C $C^{\text {hi }}$ monocytes in the blood of control and splenectomized $m d x(n=13, m d x$ control; $n=10, m d x$ splenectomized) and (E) WT mice ( $n=6$, WT control; $n=5$, WT splenectomized) by flow cytometry. (F) Histogram overlay of CCR2 expression by circulating Ly6C ${ }^{\text {hi }}$ monocytes analyzed by flow cytometry. Data are presented as mean \pm SEM. ${ }^{*} P<0.05 ;{ }^{*} P<0.01$ by Student's $t$ test.

reduced at 4 and 8 weeks of age (Figure 4, C and D). There was no significant difference in the number of infiltrating $\mathrm{CD} 45^{+}$cells at 12 weeks of age, when muscle inflammation was minimal (Figure 4E). Although the kinetics of $\mathrm{CD} 45^{+}$cell infiltration of dystrophic muscle did not change, the number was consistently lower in splenectomized versus control $m d x$ mice (Figure $4 \mathrm{~F}$ ). In addition, the number of neutrophils was also significantly reduced at 4 and 8 weeks of age in splenectomized $m d x$ muscle (Figure $4, \mathrm{G}$ and $\mathrm{H}$ ), most likely because of the reduced local production of GM-CSF, as shown in Figure 2I. There was no significant difference in the number of $\mathrm{T}$ cells recruited to dystrophic muscle at 4 and 8 weeks of age in splenectomized compared with control $m d x$ mice (Figure 4 , I and J), suggesting thats, in the absence of the spleen, $\mathrm{T}$ cells are recruited from regional lymph nodes. Finally, the lack of $\mathrm{Ly} 6 \mathrm{C}^{\mathrm{hi}}$ monocytes ameliorated early dystrophic muscle necrosis at 4 weeks (Figure 4, $\mathrm{K}$ and L).

These findings suggest that splenic Ly6 $\mathrm{C}^{\text {hi }}$ monocytes contribute to dystrophic muscle inflammation and early muscle fiber damage.

Removal of the splenic reservoir of $L y 6 C^{\text {hi }}$ monocytes improves early regeneration but hinders later stage muscle fiber repair. Monocytes recruited to sites of tissue injury perform diverse effector functions, ranging from proinflammatory cytokine production and amplification of the immune response to clearance of dead cells and stimulating angiogenesis and regeneration $(7,14)$. We therefore wondered whether the absence of splenic Ly6 $\mathrm{C}^{\text {hi }}$ monocytes might affect the ability of dystrophic muscle to regenerate. In the $m d x$ mouse model, the acute inflammatory phase in limb muscle begins at 2 weeks of age and is characterized by a peak in inflammation at 4 weeks, after which inflammation subsides and returns to almost basal level by 12 weeks of age $(4,6)$. Throughout this time, regeneration is ongoing and reaches a peak as inflammation begins to wane. We found that the frequency of embryonic myosin heavy chain ${ }^{+}\left(\mathrm{eMHC}^{+}\right)$ regenerating fibers in limb muscle of splenectomized $m d x$ mice was significantly increased compared with controls at 4 weeks of age (Figure 5, A and B). At this stage, there was no difference in the size (Figure 5C) or mean cross-sectional area (CSA) of regenerating fibers (Supplemental Figure 5E). In support of these data, the cytokine typically associated with regeneration, IL-10, was significantly increased in limb muscle from splenectomized $m d x$ mice compared with controls at 4 weeks of age (Supplemental Figure 4E). These results suggest that, in the absence of splenic Ly6 $\mathrm{C}^{\text {hi }}$ monocytes, regeneration is 
augmented in dystrophic muscle during the early phase of the pathology, at 4 weeks. This increase in regeneration was not due to increased damage, because muscle necrosis and inflammation were reduced in splenectomized $m d x$ mice (Figure $4, \mathrm{~K}$ and $\mathrm{L}$ ). Another possible reason for the boost in early regeneration following splenectomy could be increased activation of satellite cells. However, we found no significant difference in the level of myogenic markers, including Pax7, MyoD, and myogenin mRNA, in muscle from control and splenectomized $m d x$ mice (Supplemental Figure 6A). While the regenerating area remained slightly increased at 8 weeks compared with that of controls (Supplemental Figure 5C), the number of small regenerating fibers $(\mathrm{CSA}<500 \mu \mathrm{m})$ remained high in limb muscle of splenectomized $m d x$ mice, whereas the number of bigger fibers (CSA 1000-2000 $\mu \mathrm{m}$ ) was reduced compared with that of controls (Figure 5D). There was no significant difference in the average CSA of regenerating fibers between splenectomized and control $m d x$ mice at 8 weeks (Supplemental Figure 5F). During the later phase of the disease, at 12 weeks of age, regeneration was reduced (Supplemental Figure 5D), and the size of regenerating fibers remained smaller in the muscle of splenectomized $m d x$ mice compared with that of controls (Figure 5E). Interestingly, the impaired muscle fiber growth and regeneration at 8 and 12 weeks of age was accompanied by increased necrosis (Supplemental Figure 5, A and B). These findings suggest that splenic Ly6 $\mathrm{C}^{\text {hi }}$ monocytes are necessary for efficient muscle regeneration in muscular dystrophy. Impaired muscle regeneration has been associated with a reduction in angiogenesis and increased fibrosis (16). Indeed, the number of $\mathrm{CD} 31^{+}$capillaries per fiber was reduced in the absence of splenic Ly6 $\mathrm{C}^{\text {hi }}$ monocytes (Figure 5, F and G). Furthermore, although a histological analysis of collagen deposition in muscle following splenectomy revealed a modest increase in fibrosis in splenectomized $m d x$ mice at 12 weeks of age, the expression level of the fibrosis marker collagen 1A was significantly increased in muscles of splenectomized $m d x$ mice compared with that in controls (Supplemental Figure 7, A-C). Finally, muscle function in splenectomized $m d x$ mice was compromised, as evidenced by a significant reduction in muscle strength (Figure $5 \mathrm{H}$ ).

$A$ delay in proinflammatory to antiinflammatory macrophage shift contributes to impaired regeneration following splenectomy. Soon after reaching dystrophic muscle, Ly6 $\mathrm{C}^{\text {hi }}$ monocytes differentiate into $\mathrm{F} 4 / 80^{+} \mathrm{Ly} 6 \mathrm{C}^{\text {h }}$ proinflammatory macrophages. As inflammation progresses, a phenotypic transition begins to take place whereby proinflammatory $\mathrm{F} 4 / 80^{+} \mathrm{Ly} 6 \mathrm{C}^{\text {hi }}$ macrophages gradually lose the expression of Ly6C and upregulate the expression of $\mathrm{CD} 206$, eventually giving rise to proregenerative $\mathrm{F} 4 / 80^{+} \mathrm{Ly} 6 \mathrm{C}^{\mathrm{lo}} \mathrm{CD} 206^{+}$macrophages $(6,7,14)$. In the absence of splenic Ly6 $\mathrm{C}^{\mathrm{hi}}$ monocytes, we observed a delay in this shift in $m d x$ muscle at 12 weeks of age (Figure 6, A-C). A comparison of the forward (size) and side scatter (granularity) characteristics of $\mathrm{F} 4 / 80^{+} \mathrm{Ly} 6 \mathrm{C}^{\text {hi }} \mathrm{CD} 206^{\text {lo }}$ and $\mathrm{F} 4 / 80^{+} \mathrm{Ly} 6 \mathrm{C}^{\mathrm{lo}} \mathrm{CD} 206^{+}$macrophages revealed that they are of a similar size but were more granular in muscle from control compared with splenectomized $m d x$ mice, suggesting differences in cytoplasmic complexity (Figure 6D). Finally, as mentioned above, as Ly6C $\mathrm{C}^{\text {hi }}$ macrophages lose the expression of Ly6C, they upregulate the expression of CD206, a marker of antiinflammatory macrophages. As illustrated in Figure 6E, while Ly6 $\mathrm{C}^{\mathrm{lo}}$ macrophages in control $m d x$ muscle gradually increased the expression of CD206 over the course of the disease, this increase was not observed in Ly6C $\mathrm{C}^{\text {lo }}$ macrophages in splenectomized $m d x$ muscle (Figure $6 \mathrm{E}$ ). The expression level of IL-1 $\beta$, a proinflammatory cytokine produced by activated $\mathrm{Mo} / \mathrm{MP}$, was significantly increased in muscle of splenectomized $m d x$ mice compared with controls (Supplemental Figure 4C), reflecting the delay in the shift from proinflammatory to antiinflammatory macrophages. These findings suggest that one of the ways by which lack of splenic Ly6 $\mathrm{C}^{\mathrm{hi}}$ monocytes impedes dystrophic muscle regeneration is by causing a delay in the phenotypic shift from proinflammatory to proregenerative macrophages, thereby altering the tissue cytokine environment in ways that do not support adequate muscle fiber growth.

\section{Discussion}

Inflammatory Ly6 $\mathrm{C}^{\mathrm{hi}}$ monocytes are the principal effector cells in dystrophic muscle pathology $(2,6$, 14). The origin of these cells in dystrophic muscle has not been directly studied before, and the general assumption has been that they originate from the bone marrow. However, following the identification of the spleen as an alternative source of Ly6 $\mathrm{C}^{\mathrm{hi}}$ monocytes by Swirski et al. (10), a number of studies have emphasized the critical role of the spleen as an emergency reservoir from which these cells are deployed to sites of inflammation, as shown during ischemic myocardial injury and atherosclerosis $(12,13,17)$. Here, we provide further evidence of the spleen as a dominant reservoir of Ly $6 C^{\text {hi }}$ monocytes in the context of chronic muscle injury in muscular dystrophy. Reminiscent of the kinetics of the 
A

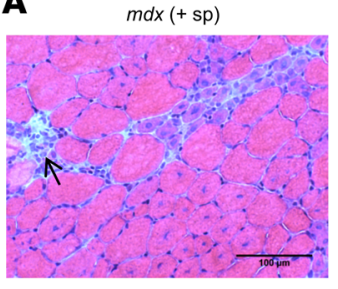

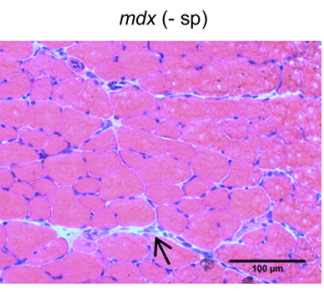

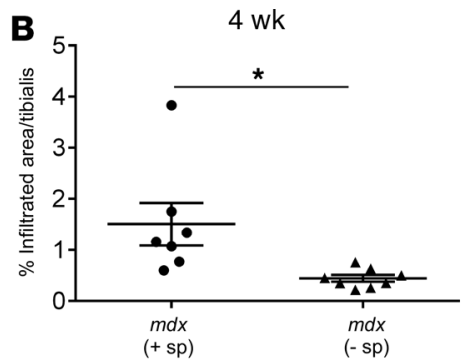

$(+\mathrm{sp})$
C

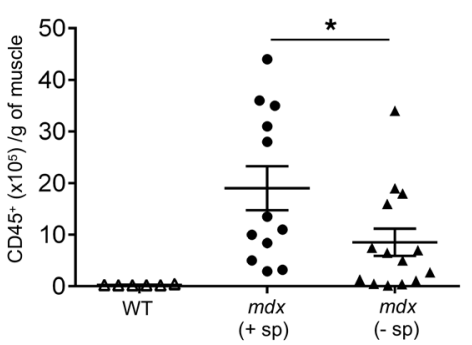

F

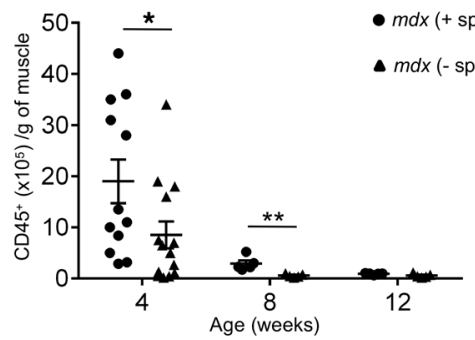

I

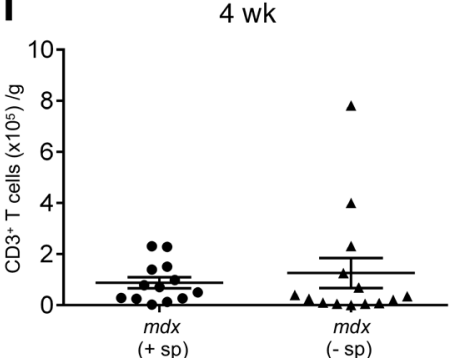

D

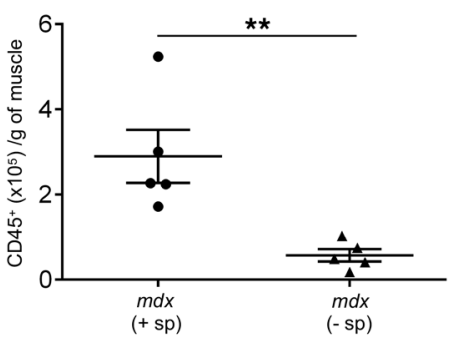

G

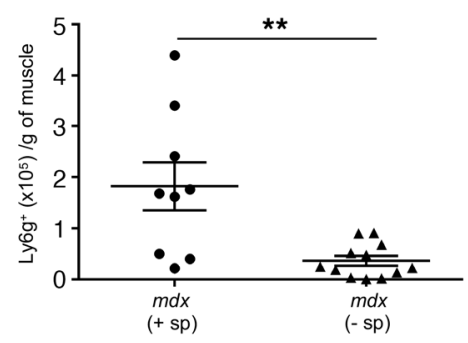

J

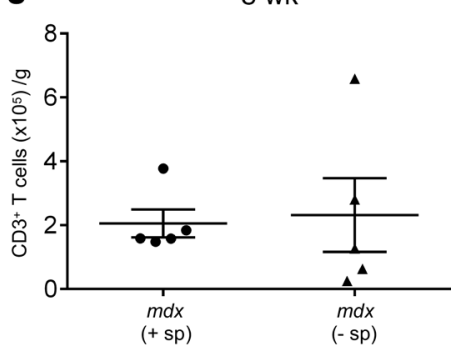

E

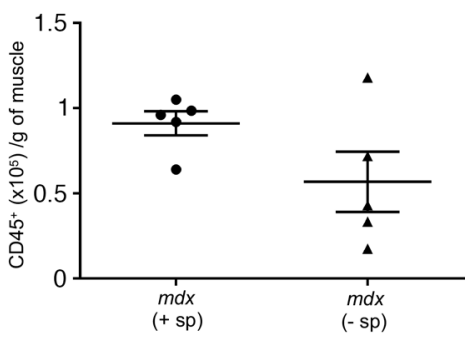

H

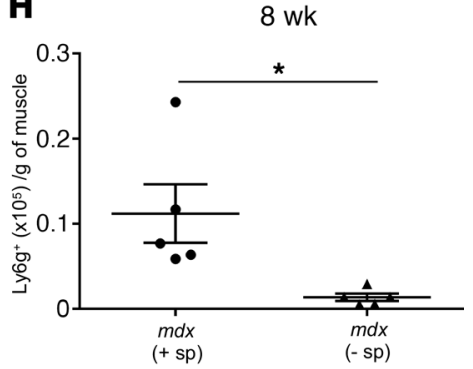

$\mathbf{K}$

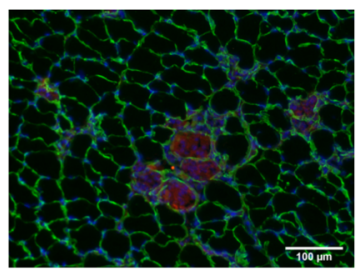

Dapi; Laminin; IgG
4 wk

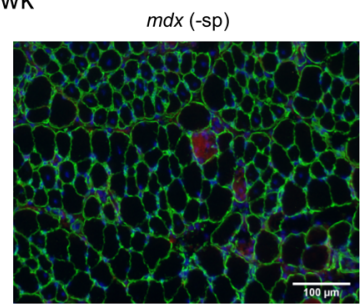

L

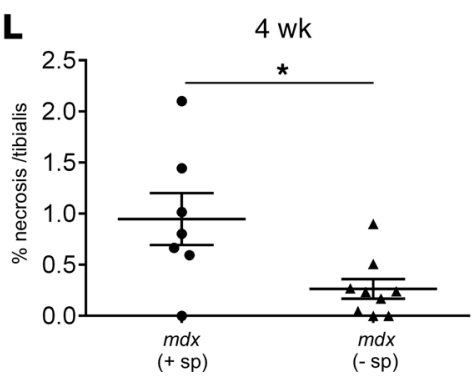

Figure 4. Splenectomy reduces dystrophic muscle inflammation and early necrosis. (A) Representative H\&E staining of tibialis anterior (TA) muscle sections showing reduced areas of infiltration (arrows) in splenectomized ( $\mathrm{sp}$ ) mdx mice compared with control (+ sp) at 4 weeks of age. Scale bars: 100 $\mu \mathrm{m}$. (B) A summary graph of histological analysis showing the percentage of infiltration area ( $n=7, m d x$ control; $n=8, m d x$ splenectomized). Following splenectomy at 2 weeks of age the inflammatory cell infiltrate in hind limb muscle was analyzed by flow cytometry at 4,8 , and 12 weeks of age (C-J). (C) Total number of $\mathrm{CD}_{4} 5^{+}$cells normalized to muscle mass in control and splenectomized $m d x$ mice at 4 weeks $(n=6, \mathrm{WT} ; n=12, m d x$ control; $n=14, m d x$ splenectomized), (D) 8 weeks ( $n=5$ for all groups), and (E) 12 weeks of age $\left(n=5\right.$ for all groups). (F) Summary graph showing the kinetics of CD45 $5^{+}$cells in muscle of control and splenectomized $m d x$ mice at 4,8 , and 12 weeks of age. (G) Total number of Ly6g' neutrophils in muscle of control and splenectomized $m d x$ mice at 4 and $(\mathbf{H}) 8$ weeks of age. (I) Total number of $C D 3^{+} T$ cells in muscle of control and splenectomized $m d x$ mice at 4 and (J) 8 weeks of age. (K) Representative TA muscle sections stained with IgG (red) to reveal necrotic muscle fibers showing reduced necrosis in muscle of splenectomized $m d x$ mice compared with control. Scale bars: $100 \mu \mathrm{m}$. (L) Quantification of necrosis ( $n=7$, control; $n=9$, splenectomized $m d x$ mice) at 4 weeks. Data are presented as mean \pm SEM. ${ }^{*} P<0.05 ;{ }^{*} P<0.01$ by Student's $t$ test $(\mathbf{B}, \mathbf{D}, \mathbf{G}, \mathbf{H}$, and $\mathbf{L}$ ) and 1 -way ANOVA (C and $\mathbf{F}$ ). 
A

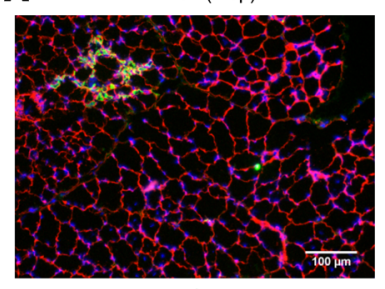

Dapi; Laminin; eMHC
C

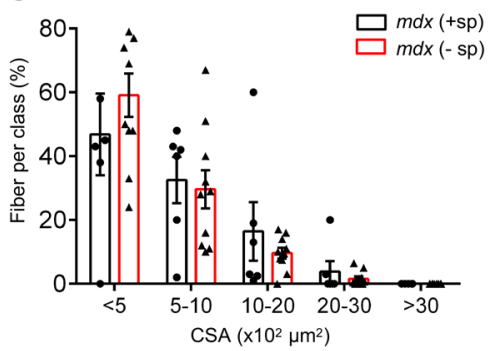

$m d x(-\mathrm{sp})$

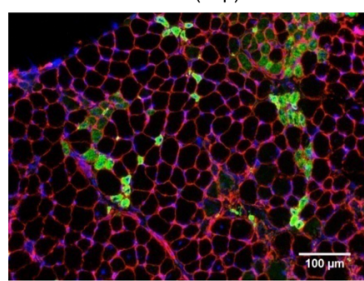

D

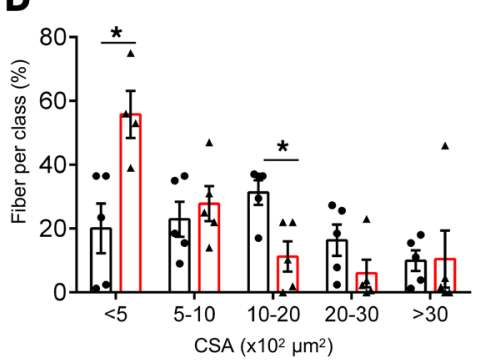

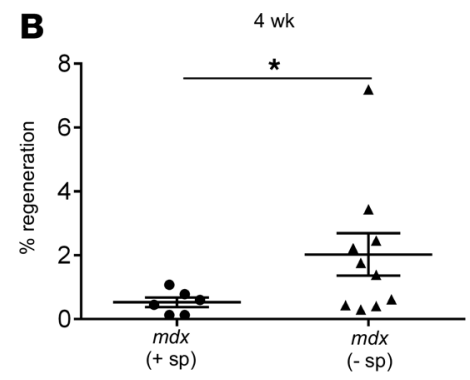

E

12 wk

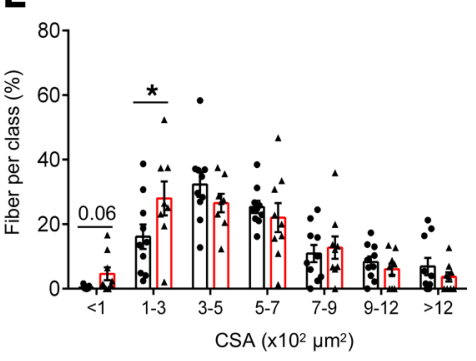

$\mathbf{F}$

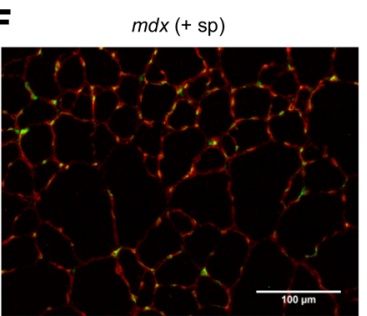

Laminin; CD31

H

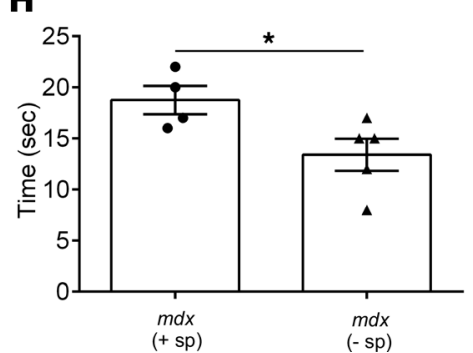

$m d x(-\mathrm{sp})$

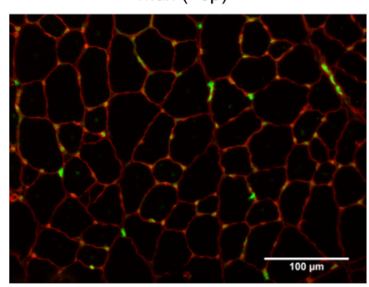

$\longdiv { 1 0 0 \mathrm { um } = }$

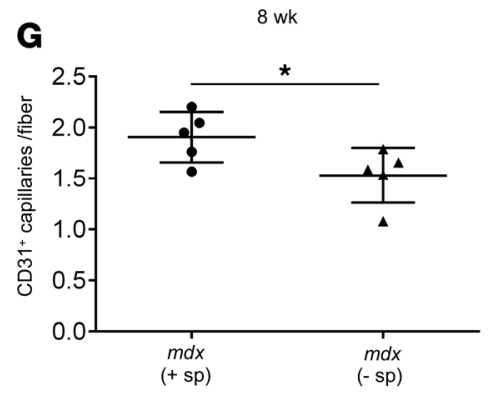

Figure 5. Splenic Ly6 $\mathrm{C}^{\mathrm{hi}}$ monocytes are necessary for efficient repair of dystrophic muscle fibers. (A) Representative TA muscle sections stained for embryonic myosin heavy chain (eMHC) to visualize regenerating muscle fibers (green) showing an increase in eMHC+ fibers in splenectomized (- $\mathrm{sp}$ ) compared with control ( + sp) $m d x$ mice at 4 weeks of age during the early phase of the pathology. Scale bars: $100 \mu \mathrm{m}$. (B) Summary graph showing the percentage of regenerating area $(n=6, m d x$ control; $n=10 \mathrm{mdx}$ splenectomized). (C) Regenerating fiber size frequency distribution determined by measuring the cross-sectional area (CSA) of regenerating fibers in TA muscle from control and splenectomized mice at 4 weeks of age ( $n=6, m d x$ control; $n=10 \mathrm{mdx}$ splenectomized), (D) 8 weeks of age ( $n=5$ for both groups), and (E) 12 weeks of age ( $n=10, m d x$ control; $n=9$ mdx splenectomized). (F) Representative TA muscle sections stained for CD31 to reveal capillaries (green). Scale bars: $100 \mu \mathrm{m}$. (C) Number of CD31+ capillaries per muscle fiber at 8 weeks of age in muscle of control and splenectomized $m d x$ mice. (H) A graph showing a reduction in muscle strength in splenectomized compared with control mdx mice at 12 weeks of age, measured by a hanging wire test, which determines the time it takes for a mouse to fall off a metal wire following exhaustion. Data are presented as mean \pm SEM. ${ }^{*} P<0.05$ by Student's $t$ test.

inflammatory response in dystrophic muscle (6), Ly6 $\mathrm{C}^{\text {hi }}$ monocytes in the spleen showed the first signs of expansion early, before the onset of overt disease, and remained elevated until the end of the acute inflammatory phase. This early expansion was accompanied by an increase in the expression of CCR2, a chemokine receptor known to be necessary for monocyte egress. Such an early response in the spleen has also been observed in a mouse model of ALS, where similar to our findings, Ly6 $\mathrm{C}^{\mathrm{hi}}$ monocytes in the spleen upregulated CCR2 expression prior to disease onset (11). Interestingly, these early events in the spleens of $m d x$ mice coincided with an increase in limb muscle of CCL2, the ligand for CCR2, 
A
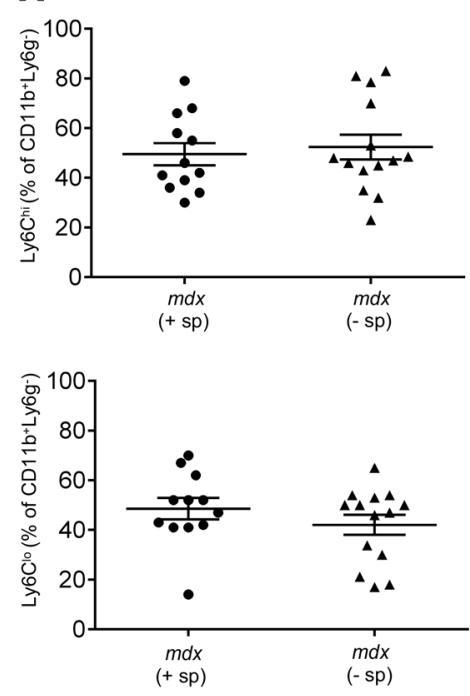

D
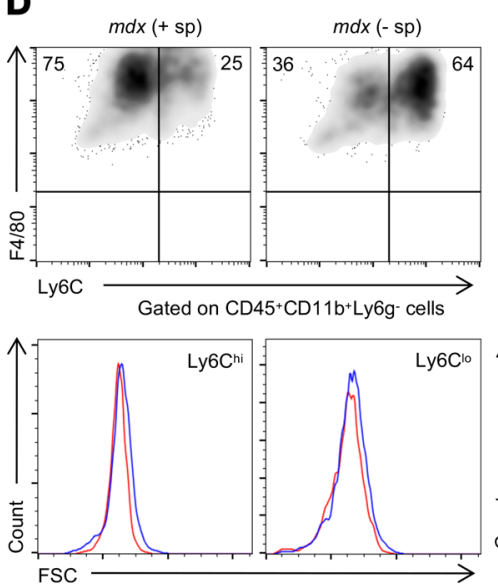

B
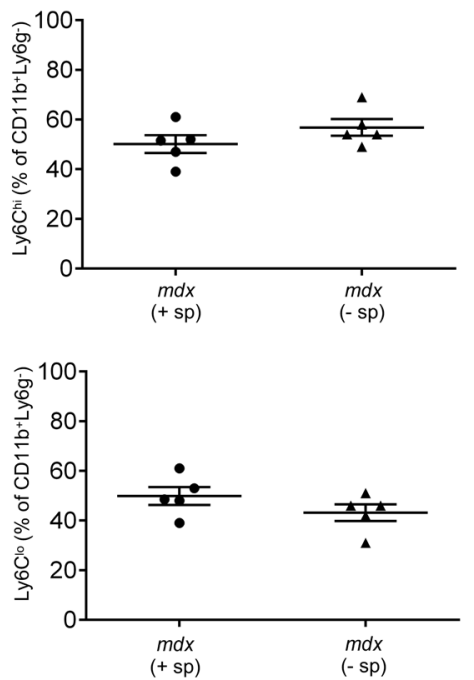

$12 \mathrm{wk}$
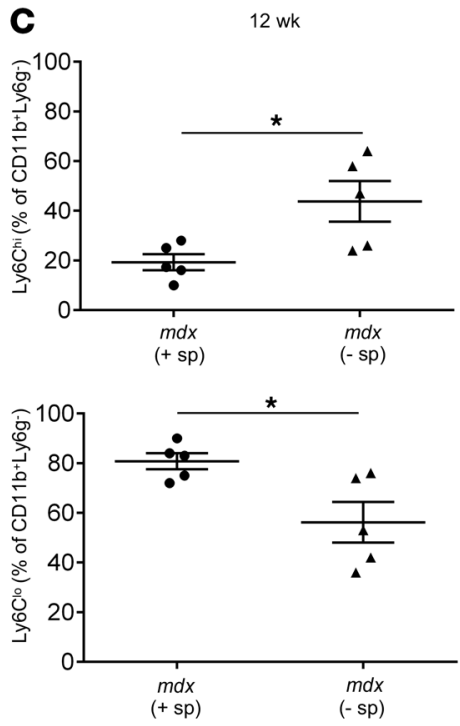
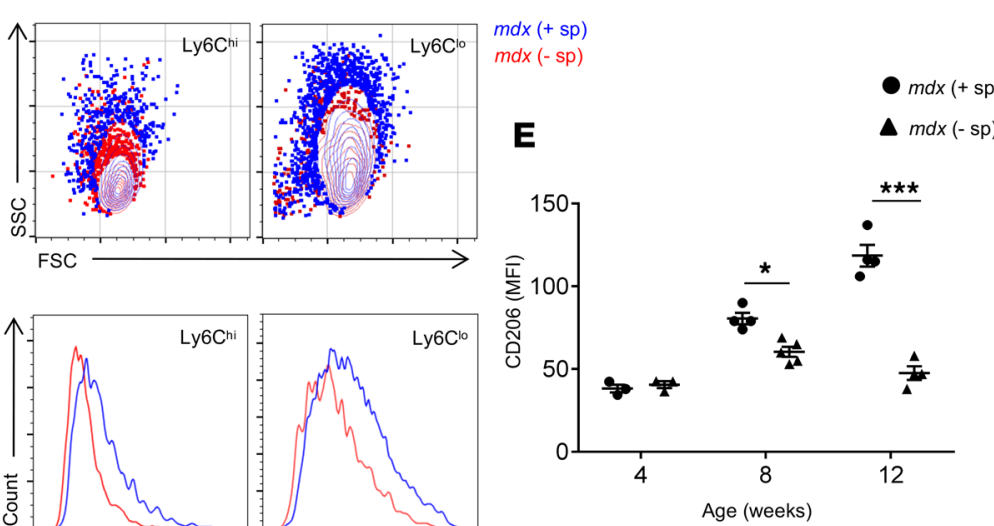

ssc

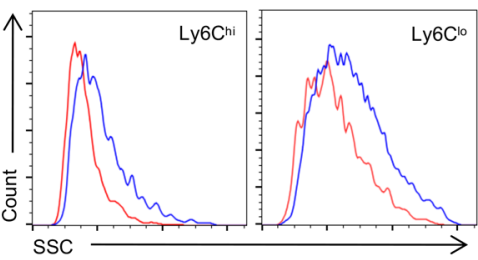

Figure 6. Splenectomy delays the phenotypic switch from proinflammatory Ly6 $\mathrm{C}^{\mathrm{hi}}$ to proregenerative Ly $6 \mathrm{C}^{10}$ macrophages in dystrophic limb muscle. (A) Frequency of Ly6Chi and Ly6C ${ }^{10}$ macrophages in $m d x$ control and splenectomized muscle at $4(n=12$, control and $n=14$, splenectomized), (B) 8 ( $n=5$ for both groups), and (C) 12 ( $n=5$ for both groups) weeks of age analyzed by flow cytometry. (D) Representative density and contour flow cytometry plots and histogram overlays of forward and side scatter parameters of Ly6Chi and Ly6 $\mathrm{C}^{\mathrm{IO}}$ macrophages in control and splenectomized mdx muscle. (E) Kinetics of CD206 expression on Ly6C $C^{10}$ macrophages assessed by flow cytometry. Shown is the mean fluorescence intensity (MFI) of CD206 expression by Ly6C ${ }^{10}$ macrophages in muscle from control and splenectomized $m d x$ mice at 4 ( $n=3$ for both groups), $8(n=4, m d x$ control; $n=5, m d x$ splenectomized), and 12 weeks of age ( $n=4$ for both groups). Data are presented as mean $\pm \mathrm{SEM}^{*} P<0.05,{ }^{* * *} P<0.001$ by Student's $t$ test.

which mediates monocyte recruitment, and of GM-CSF, a cytokine, which in Ly6 $\mathrm{Ch}^{\text {hi }}$ monocytes stimulates chemotaxis, phagocytosis, and induction of genes linked to inflammasome function (15). It is possible that GM-CSF produced by activated macrophages and fibroblasts in dystrophic muscle stimulate myelopoiesis in distal sites such as the spleen and the bone marrow, as recently shown in acute myocardial ischemia (18). Nevertheless, unlike in the spleen, we found no significant increase in the number of Ly6 $\mathrm{C}^{\text {hi }}$ monocytes in the bone marrow of $m d x$ mice and no upregulation of CCR2, suggesting that the spleen represents the dominant supplier of these cells during the early stages of dystrophic muscle injury, as previously demonstrated during ischemic myocardial injury and atherosclerosis $(12,13,17)$. Thus, splenectomy performed before disease onset reduced the number of Ly6 $\mathrm{C}^{\text {hi }}$ monocytes infiltrating dystrophic limb muscle by $70 \%$ and ameliorated inflammation and necrosis during the early stages of the disease. Although the number of Ly6 $\mathrm{C}^{\text {hi }}$ monocytes increased in the bone marrow of splenectomized $m d x$ mice compared with controls, the bone marrow failed to fully compensate for the absence of the spleen, most probably due to the reduced level of the monocyte mobilizing chemokine CCL2 and the GM-CSF cytokine released from dystrophic muscle following splenectomy. The fact that these 
cytokines were reduced in dystrophic muscle after splenectomy suggests that splenic Ly6 $\mathrm{C}^{\text {hi }}$ monocytes contribute to the early muscle fiber damage and amplification of the inflammatory response.

Previous studies have shown that targeting Ly6 $\mathrm{C}^{\text {hi }}$ monocytes through CCR2 or treatment with antiLy6C mAb reduces dystrophic muscle damage. Mojumdar et al. demonstrated that CCR2 deficiency in $m d x$ mice reduces the number of Ly6 $\mathrm{C}^{\text {hi }}$ monocytes recruited to the diaphragm and ameliorates diaphragm muscle degeneration during the early stages of the disease (19). Interestingly, the authors found that splenectomy had no effect on the number of Ly $6 \mathrm{C}^{\text {hi }}$ monocytes recruited to the diaphragm. The reason for the different outcomes between this and our study could be that the focus was on different tissues (diaphragm versus limb muscle) with different inflammation kinetics. For example, in our study we performed splenectomy at 2 weeks of age because our kinetics data showed that to be the earliest time when monocytes begin to infiltrate limb muscle. We sacrificed the animals 2 weeks later in order to examine the effect on the peak of inflammation and 6 and 10 weeks later in order to study regeneration. Although much less striking, the difference in the number of Ly6 $\mathrm{C}^{\text {hi }} \mathrm{Mo} / \mathrm{MP}$ observed at 4 weeks was still present at 8 weeks but was absent at 12 weeks of age. Mojumdar et al. splenectomized the animals at 3 weeks of age and examined the diaphragm 6 weeks later when inflammation likely begins to subside and any differences in numbers that might have existed become less obvious (19).

A more recent study, using $m d x^{5 c v}-C C R 2^{-/-}$mice also showed a beneficial effect on muscle pathology by reducing the number of infiltrating Ly $6 \mathrm{C}^{\text {hi }}$ monocytes. However, the improvement in diaphragm and limb muscle damage and fibrosis observed at 14 weeks was not sustained at 6 months (20). Similar to this study, we found that the beneficial effect of splenectomy observed during the early phase was not sustained during the later stages of the disease. While lack of splenic monocytes reduced inflammation and necrosis and ameliorated regeneration early in the acute disease phase, a defect in muscle fiber repair became evident by 8 weeks and was still apparent at 12 weeks. Smaller regenerating fibers predominated in splenectomized $m d x$ mice, and there was a reduced appearance of bigger fibers, suggesting impaired muscle fiber growth or maturation. This compromised repair of muscle fibers, together with the reduced number of $\mathrm{CD} 1^{+}$capillaries per fiber, led to a reduction in muscle strength, suggesting that in the absence of splenic Ly $6 \mathrm{C}^{\text {hi }}$ monocytes the dystrophic phenotype worsens. Removal of the splenic reservoir of Ly $6 \mathrm{C}^{\text {hi }}$ monocytes did not affect the ability of satellite cells to undergo activation, and the impairment in muscle fiber growth is likely due to the delay in the appearance of the proregenerative $\mathrm{F} 4 / 80^{+} \mathrm{Ly} 6 \mathrm{C}^{\mathrm{lo}} \mathrm{CD} 206^{+}$ macrophage subset and the consequent changes in the tissue cytokine environment that do not adequately support muscle fiber growth, as previously proposed in various contexts of muscle injury $(14,21,22)$. On the other hand, the balance of proinflammatory Ly $6 \mathrm{C}^{\text {hi }}$ to proregenerative Ly $6 \mathrm{C}^{\text {lo }}$ macrophages in splenectomized $m d x$ muscle was not affected during the early phase of the disease, which, together with the higher level of proregenerative cytokine IL-10 and the decrease in inflammation might have contributed toward the increase in early regeneration we observed at 4 weeks of age.

Given that Mo/MP perform diverse effector functions both in the context of tissue injury and regeneration, these findings were not surprising. The optimal response to tissue injury depends on a fine balance between inflammatory monocyte numbers and their phenotype. Recruitment of too few or too many is likely to create an unfavorable environment for the resolution of tissue inflammation and compromise the reestablishment of tissue homeostasis. Thus, it seems probable that the $70 \%$ reduction in the number of Ly6 $\mathrm{C}^{\mathrm{hi}}$ monocytes in dystrophic muscle following splenectomy precluded the effective clearance of necrotic cells and debris, eventually hindering repair and worsening fibrosis. It is also possible that the spleen provides a distinct monocyte subtype or subtypes that differentially participate in the inflammatory and regenerative phase of dystrophic muscle injury and are recruited as part of separate waves. Future studies are needed to test these possibilities and broaden our understanding of the origin and function of monocytes and macrophages in muscular dystrophy.

Finally, we cannot exclude the possibility that absence of cells other than monocytes recruited from the spleen might have contributed to these outcomes. Nevertheless, we found no difference in the number of $\mathrm{CD}^{+} \mathrm{T}$ cells recruited to muscle of splenectomized animals, and although we did not examine regulatory $\mathrm{T}$ cells, we found similar levels of the immunoregulatory cytokines IL-10 and TGF- $\beta$ in muscle of splenectomized versus control $m d x$ mice.

In conclusion, we show that the spleen is an indispensable source of Ly6 $\mathrm{C}^{\mathrm{hi}}$ monocytes in muscular dystrophy and that splenic monocytes contribute to dystrophic muscle injury early in the course of the disease but are also necessary for effective regeneration during the later stages of the pathology. 
An important question to be addressed in the future is whether the spleen is just an extramedullary site to which the bone marrow outsources the production of $\mathrm{Ly} 6 \mathrm{C}^{\text {hi }}$ monocytes during chronic inflammation or whether it also represents an important monocyte training ground.

The present study underscores the complexity of monocyte responses in tissue injury and highlights the need for a greater understanding of the origin-, time-, and context-dependent effector functions of these cells. Only then, will we be able to design novel pharmaceuticals to target monocytes in muscular dystrophy in a way that will reduce damage without compromising regeneration.

\section{Methods}

Mice. C57BL/10ScSn-Dmd ${ }^{m d x}$ and C57BL/10ScSn mice were purchased from The Jackson Laboratory. Only males were used.

Cell isolation and processing. Muscle single-cell suspensions were obtained as previously described (6). Briefly, mice were perfused with PBS, and hind limb musculature (tibialis anterior, gastrocnemius, and quadriceps) was excised, weighed, cut into $1-\mathrm{mm}$ pieces, and digested with $1 \mathrm{mg} / \mathrm{ml}$ Collagenase type 4 (Worthington) for 1.5 hours in a prewarmed $\left(37^{\circ} \mathrm{C}\right)$ water bath with agitation. Hind limb muscle from each mouse was processed individually. The digested muscle preparation was first passed through a $70-\mu \mathrm{m}$ cell strainer (BD Falcon) and then through a $40-\mu \mathrm{m}$ cell strainer (BD Falcon), washed in DMEM/10\% FBS, and resuspended in FACS buffer (PBS 1\% FBS). Single cells were counted with a hemocytometer and analyzed by flow cytometry.

Splenocytes were obtained by grinding the spleen between the rough edges of 2 microscopy slides. Blood was collected by cardiac puncture. Following the lysis of RBCs, cells were counted, stained with the relevant antibodies, and analyzed by FACS.

Splenectomy. Splenectomy was performed on 2-week-old $m d x$ or WT mice anesthetized with zoletil (Virbac Laboratories) (50:50 mg/ml) and rompun (Bayer) $(20 \mathrm{mg} / \mathrm{ml})$. After opening of the abdominal cavity, the spleen vessels were cauterized and the spleen was carefully excised. Control animals underwent anesthesia and opening of the abdominal cavity but without removal of the spleen. Animals were sacrificed 2, 6, or 10 weeks after splenectomy for analysis.

Flow cytometry. Prior to staining, single-cell suspensions were incubated with anti-CD16/32 (Biolegend, clone 93, $1 \mu 1 /$ test) to block nonspecific binding to Fc receptors. Samples were stained with the relevant antibodies listed in Supplemental Table 1. Cell viability was assessed with DAPI (Biolegend). Compensation was set using the ABC compensation beads kit (Thermo Fisher). Samples were acquired with a CyAn $\mathrm{ADP}$ (DAKO), and acquired data were analyzed using FlowJo software version 10.

Histology. Tibialis muscles were embedded in Tissue freezing medium (Leica) and snap frozen in liquid nitrogen-cooled isopentane. Frozen muscles were cut into $8-\mu \mathrm{m}$ sections and stored at $-20^{\circ} \mathrm{C}$ until use. For morphological assessment, frozen sections were stained with H\&E. The extent of necrosis was determined by staining with fluorescently labeled IgG (MilliporeSigma, 1:100). Regeneration was assessed by staining for eMHC (Hybridoma Bank, clone F1.652, 1:10). The CSA of regenerating fibers was examined using anti-laminin antibody (MilliporeSigma, 1:200) to visualize the fibers and ImageJ software (NIH) for calculating the area of regeneration (23). Angiogenesis was examined by staining of muscle sections with CD31 antibody (Biolegend, 1:100) to visualize the capillaries as previously described (23). All analyses were done in blinded fashion and on 3 muscle sections per mouse. Fibrosis was assessed by staining of muscle sections with Sirius red (MilliporeSigma) to visualize areas of collagen deposition and quantified using ImageJ software (NIH).

Hanging wire test. Each mouse was placed on top of a wire lid from a typical housing cage. The lid was then turned upside down with the mouse hanging by its fore limbs, and the time until fall was recorded. Each mouse was given 3 attempts to reach the 180-second limit before releasing its grip and falling, with a 30 -second recovery period prior to the next attempt. The longest suspension time was recorded.

Quantitative real-time PCR. RNA was isolated from muscle single cells using Trizol (MilliporeSigma) according to the manufacturer's instructions. cDNA was synthesized using the High-Capacity cDNA Reverse Transcription kit (Applied Biosystems). PCR amplification was performed using the SensiMix SYBR Low-ROX Kit (Bioline), following the manufacturer's protocol. Data analysis was performed using 7500 Software v2.0.6 (Applied Biosystems). Data are expressed as fold change in expression levels. Relative expression levels were normalized to GAPDH mRNA. The primer pairs used for amplification are listed in the Supplemental Methods. 
Statistics. All statistical analyses were performed using GraphPad Prism software version 6. Data are presented as mean \pm SEM. Statistical significance was determined using unpaired 2-tailed Student's $t$ test with Welch's correction for unequal variances or 1-way ANOVA followed by Sidak's correction for multiple comparisons as appropriate. A $P$ value of less than or equal to 0.05 was considered statistically significant.

Study approval. All mice were bred and housed in the Sapienza University of Rome pathogen-free Histology Department-accredited animal facility. All procedures involving mice were approved by the Italian Ministry for Health and were conducted according to the NIH guidelines and Directive 2010/63/EU.

\section{Author contributions}

BLO conceived the study, designed the experiments, and analyzed and interpreted data. GR and RDM performed the experiments and analyzed data. AB and JM helped with sample collection and histological analysis. MB contributed to experimental design and data interpretation and provided resources and financial support. BLO sourced funding for the study and wrote the manuscript. All authors critically read, edited, and approved the final manuscript.

\section{Acknowledgments}

This work was supported by a grant from the Dutch Duchenne Parent Project, the Netherlands, to BLO, and benefited from research grants from Parent Project Italy and University of Rome to MB.

Address correspondence to: Biliana Lozanoska-Ochser, Via A. Scarpa 14, Rome 00161, Italy. Phone: 39.064.976.6573; Email: biliana.lozanoska-ochser@uniroma1.it.

GR's present address is: Comprehensive Heart Failure Center, University Hospital Würzburg, Würzburg Germany.

1. Emery AE. The muscular dystrophies. Lancet. 2002;359(9307):687-695.

2. Rosenberg AS, et al. Immune-mediated pathology in Duchenne muscular dystrophy. Sci Transl Med. 2015;7(299):299rv4

3. Evans NP, Misyak SA, Robertson JL, Bassaganya-Riera J, Grange RW. Immune-mediated mechanisms potentially regulate the disease time-course of Duchenne muscular dystrophy and provide targets for therapeutic intervention. PM R. 2009;1(8):755-768.

4. Spencer MJ, Tidball JG. Do immune cells promote the pathology of dystrophin-deficient myopathies? Neuromuscul Disord. 2001;11(6-7):556-564.

5. Villalta SA, Rosenberg AS, Bluestone JA. The immune system in Duchenne muscular dystrophy: Friend or foe. Rare Dis. 2015;3(1):e1010966.

6. Lozanoska-Ochser B, et al. Targeting early PKC $\theta$-dependent T-cell infiltration of dystrophic muscle reduces disease severity in a mouse model of muscular dystrophy. J Pathol. 2018;244(3):323-333.

7. Guilliams M, Mildner A, Yona S. Developmental and functional heterogeneity of monocytes. Immunity. 2018;49(4):595-613.

8. Ingersoll MA, Platt AM, Potteaux S, Randolph GJ. Monocyte trafficking in acute and chronic inflammation. Trends Immunol. 2011;32(10):470-477.

9. Guilliams M, Scott CL. Does niche competition determine the origin of tissue-resident macrophages? Nat Rev Immunol. 2017;17(7):451-460.

10. Swirski FK, et al. Identification of splenic reservoir monocytes and their deployment to inflammatory sites. Science. 2009;325(5940):612-616.

11. Butovsky O, et al. Modulating inflammatory monocytes with a unique microRNA gene signature ameliorates murine ALS J Clin Invest. 2012;122(9):3063-3087.

12. Leuschner F, et al. Rapid monocyte kinetics in acute myocardial infarction are sustained by extramedullary monocytopoiesis. J Exp Med. 2012;209(1):123-137.

13. Robbins CS, et al. Extramedullary hematopoiesis generates Ly-6C(high) monocytes that infiltrate atherosclerotic lesions. Circulation. 2012;125(2):364-374.

14. Tidball JG. Regulation of muscle growth and regeneration by the immune system. Nat Rev Immunol. 2017;17(3):165-178.

15. Becher B, Tugues S, Greter M. GM-CSF: from growth factor to central mediator of tissue inflammation. Immunity. 2016;45(5):963-973.

16. Buckley AF, Bossen EH. Skeletal muscle microvasculature in the diagnosis of neuromuscular disease. J Neuropathol Exp Neurol. 2013;72(10):906-918

17. Hulsmans M, et al. Cardiac macrophages promote diastolic dysfunction. J Exp Med. 2018;215(2):423-440.

18. Anzai A, et al. The infarcted myocardium solicits GM-CSF for the detrimental oversupply of inflammatory leukocytes. $J$ Exp Med. 2017;214(11):3293-3310

19. Mojumdar K, et al. Inflammatory monocytes promote progression of Duchenne muscular dystrophy and can be therapeutically targeted via CCR2. EMBO Mol Med. 2014;6(11):1476-1492.

20. Zhao W, Wang X, Ransohoff RM, Zhou L. CCR2 deficiency does not provide sustained improvement of muscular dystrophy in mdx5cv mice. FASEB J. 2017;31(1):35-46 
21. Wang H, Melton DW, Porter L, Sarwar ZU, McManus LM, Shireman PK. Altered macrophage phenotype transition impairs skeletal muscle regeneration. Am J Pathol. 2014;184(4):1167-1184.

22. Jin RM, Warunek J, Wohlfert EA. Chronic infection stunts macrophage heterogeneity and disrupts immune-mediated myogenesis. JCI Insight. 2018;3(18):121549.

23. Marrocco V, et al. Pharmacological inhibition of PKC $\theta$ counteracts muscle disease in a mouse model of Duchenne muscular dystrophy. EBioMedicine. 2017;16:150-161. 